\section{$\underset{\substack{\text { hommes } \\ \text { \& migrations }}}{ }$}

\section{Hommes \& migrations}

Revue française de référence sur les dynamiques

migratoires

$1305 \mid 2014$

L'exil chilien en France

\title{
Entre histoire et mémoire
}

Le Musée de la mémoire de Santiago du Chili

\section{Bernardo Toro}

\section{OpenEdition \\ 1 Journals}

\section{Édition électronique}

URL : http://journals.openedition.org/hommesmigrations/2766

DOI : 10.4000/hommesmigrations.2766

ISSN : 2262-3353

\section{Éditeur}

Musée national de l'histoire de l'immigration

\section{Édition imprimée}

Date de publication : 1 janvier 2014

Pagination : 173-175

ISBN : 978-2919-040261

ISSN : $1142-852 X$

\section{Référence électronique}

Bernardo Toro, «Entre histoire et mémoire », Hommes \& migrations [En ligne], 1305 | 2014, mis en ligne le 11 juillet 2014, consulté le 22 septembre 2020. URL : http://journals.openedition.org/

hommesmigrations/2766 ; DOI : https://doi.org/10.4000/hommesmigrations.2766 


\section{ENTRE HISTOIRE ET MÉMOIRE LE MUSÉE DE LA MÉMOIRE DE SANTIAGO DU CHILI}

BERNARDO TORO, écrivain, rédacteur en chef de la revue Rue Saint-Ambroise

\section{Un musée contre l'oubli}

Inauguré en 2010 par Michelle Bachelet, le Musée de la mémoire et des droits de l'homme est un espace consacré aux violations des droits de I'homme commises par l'État chilien entre 1973 et 1990. À travers des archives, des documentaires, des photos, des témoignages, le musée retrace ces années parmi les plus sombres de l'histoire chilienne, dans le but de stimuler la réflexion sur l'importance du respect et de la tolérance afin que ces faits ne se reproduisent plus. Comme tous les lieux de mémoire, ce musée est né d'un refus de mémoire. Au Chili, ce refus ne concerne pas seulement les nouvelles générations désireuses de construire leur propre histoire, mais se trouve à la base des crimes perpétrés. L'effacement programmé des traces et des corps opéré par les militaires visait précisément à rendre impossible tout travail de mémoire. II a donc fallu retrouver les traces, rassembler les témoignages et rétablir les faits grâce à deux commissions d'enquête (Rettig, 1990, et Valech, 2003), avant de songer à leur consacrer un musée. Les proportions imposantes du musée semblent à la mesure de l'impressionnant désir d'occultation de la police politique. À la volonté d'occulter puis de minimiser les crimes répond l'hypertrophie de cette imposante structure de béton et de verre de $5600 \mathrm{~m}^{2}$ dressée en plein cœur de Santiago, l'opacité des pratiques répressives trouvant dans la transparence du verre une mise en lumière tardive mais définitive. Au Chili, la constitution de lieux de mémoire évoquant la répression militaire est un fait relativement récent. Suite au rapport de la commission Valech qui a reconnu pour la première fois les victimes de la torture, sept sites où la répression s'est exercée ont été déclarés "monuments nationaux". À la différence des autres lieux de mémoire, le Musée de la mémoire est un lieu d'exposition créé de toutes pièces qui ne se contente pas de rappeler les faits, il cherche aussi à produire un récit capable de véhiculer un sens et de frapper les imaginations. C'est l'un de ses aspects les plus intéressants mais aussi l'un des plus polémiques. La construction sociale de la mémoire se heurte à l'émiettement des mémoires individuelles qui sont non seulement différentes, mais souvent divergentes, voire contradictoires.

\section{Les raisons d'une polémique}

Comme son nom l'indique, ce musée est consacré à la mémoire et non à l'Histoire. Cette distinction en apparence formelle a pris une importance déterminante lors des controverses qu'il a suscitées au moment de sa création. On a, en effet, reproché au musée sa volonté d'extraire ces violations des droits de l'homme de leur contexte historique. L'option du musée de "circonscrire sa mission aux seules violations des droits de l'homme commises entre le 11 septembre 1973 et le 10 mars 1990, sans donner aux visiteurs les causes qui les ont produites, 
limite sa fonction pédagogique, selon Magdalena Krebs (chargée du patrimoine). L'absence d'un consensus historique sur le sujet, poursuit-elle, ne libère pas le musée de sa responsabilité d'offrir une vision large des faits". Une partie de l'opinion chilienne insiste sur la nécessité de replacer les crimes commis sous la dictature dans leur contexte historique (guerre froide, gouvernement marxiste, exacerbation des conflits de classes, risque de guerre civile, etc.), autant de circonstances qui, sans justifier les exactions commises les expliquent et élargissent la sphère de responsabilités. Cette opinion, qui dépasse les rangs de la droite traditionnelle, reprend à son compte la doctrine du "plus jamais", mais ajoute à la liste des faits qui ne doivent plus se reproduire l'action du gouvernement d'Allende qui, en exacerbant les conflits de classes, a mené le pays au bord de la guerre civile. Le Musée de la mémoire a été bâti contre la menace que représente un tel discours qui se sert de l'Histoire pour jeter un pont entre la responsabilité politique d'Allende et les crimes d'État de Pinochet, c'est-à-dire entre des erreurs politiques et des crimes d'État. Suivant cette même logique de contextualisation historique, les pourfendeurs du musée prétendent que les crimes perpétrés entre 1973 et 1989 constituent un épisode de violence (particulièrement sanglant, mais nullement exceptionnel) qui s'ajoute à une histoire nationale traversée de part en part par la violence sociale. Y a-t-il un musée qui commémore le massacre de Santa Maria de Iquique où sont morts près de 3000 travailleurs du salpêtre? Accorder à la violence de la dictature un caractère exceptionnel serait donc méconnaître I'histoire ou, pire, la manipuler à des fins idéologiques.

\section{Le devoir de mémoire et l'explication historique}

À cette forme de relativisme historique partagée par une partie non négligeable de la société chilienne, le musée oppose sa vocation mémo- rielle. Dans une déclaration officielle, ses responsables ont rappelé que "l'objectif du musée n'était pas de donner des informations sur les causes qui ont conduit à ces violations (...) mais de promouvoir l'idée que, quelles que soient les circonstances, ce genre de faits ne doit plus jamais se reproduire dans notre pays". Le musée obéirait ainsi à un devoir de mémoire et non à un désir d'explication historique, sa visée étant éthique et non historiographique. Si l'histoire est une opération intellectuelle et critique qui a pour but la reconstitution et la compréhension du passé, la mémoire par contre ne s'intéresse au passé que dans la mesure où il est porteur de valeurs atemporelles capables de souder une communauté. Ce que la mémoire prétend sauvegarder est moins la connaissance historique que le lien social lui-même. En l'occurrence, le Musée de la mémoire voudrait préserver la valeur "respect des droits de l'homme" de toute emprise idéologique ou scientifique qui tendrait à la relativiser.

\section{Un hiatus historique}

Cette charge apparente contre l'histoire n'est en réalité qu'une autre conception de l'histoire, qui oppose au continuum historique l'idée d'une cassure qui aurait coupé l'histoire du Chili en deux. II y aurait un avant et un après Pinochet, comme il y a un avant et un après Auschwitz.

L'enchaînement des causes et des effets propre à la dynamique historique ne saurait expliquer entièrement les crimes commis par la dictature, de la même manière que les errements de la République de Weimar ou la rigueur du traité de Versailles ne sauraient expliquer le génocide juif, l'explication poussée à son terme étant une négation de la mémoire, c'est-à-dire une menace pour le lien social. L'impératif moral qui préside à la création du musée n'est donc pas extérieur à l'histoire, mais suppose la reconnaissance d'un hiatus historique (mince mais irréductible) qu'aucune explication ne doit résorber. En un sens, le 
musée demande à la rationalité historique de reconnaître ce hiatus pour que la mémoire puisse exercer ses droits.

\section{Les critiques venant de la gauche}

Les critiques visant le manque de contexte historique du musée sont également venues de la gauche, notamment des associations de défense des droits de l'homme qui ont activement travaillé à la restauration de la mémoire, mais qui n'ont pas été associées à la conception du projet. Pour certaines d'entre elles, la décontextualisation et donc la dépolitisation de la violence militaire vident ces faits de leur substance et transforment le récit de cette époque en un film dont on aurait enlevé le son et qui deviendrait de ce fait incompréhensible. Les victimes de la dictature seraient privées du contenu de leur lutte et donc réduites une nouvelle fois au silence pour n'apparaitre que comme des victimes dont on revendique une humanité vide de tout autre attribut. Seule leur vie est prise en compte au détriment de leurs idées, alors que, paradoxalement, il s'agit de gens qui ont donné leur vie pour leurs idées. Mais c'est sans doute le prix à payer pour que cette mémoire cesse d'être le seul patrimoine de la gauche et devienne celui de tous les Chiliens.

\section{L'exil, du lieu commun à la réalité}

Un combat reste pourtant à mener et il concerne l'exil. L'ironie morbide de Pinochet, qui parlait des décrets d'expulsion comme de bourses d'étude qu'il octroyait à des gens qui ne les méritaient pas, est aujourd'hui partagée par une bonne partie de l'opinion chilienne. L'exil reste aux yeux des Chiliens, y compris de gauche, plutôt une récompense qu'une punition. Cette idée d'un "exil doré dans les pays du Premier Monde" n'est pas le seul soupçon qui pèse sur les exilés. Certains leur reprochent aussi leur lâcheté (qui les a poussés à quitter le pays à une époque où le courage consistait à rester) et leur opportunisme (qui les a incités à rentrer pour prendre les postes que la démocratie renaissante leur offrait). Une bonne partie des personnalités des gouvernements successifs de la Concertation est composée, en effet, d'anciens exilés. Sous un mode très fantasmatique, toutes les frustrations engendrées par les vingt années de gouvernement concertationniste peuvent donc être mises sur le compte des exilés qui, après avoir joué aux héros en Europe, sont revenus au Chili pour gérer le modèle ultra-libéral imposé par la dictature. Comme les retornados le savent bien, la condition essentielle de leur réinsertion au Chili reste l'oubli des années d'exil, comme s'il s'agissait là d'une tare honteuse que les Chiliens sont prêts à tolérer à condition qu'elle soit parfaitement dissimulée. La connaissance de la réalité de l'exil, de ses difficultés, de ses zones d'ombre, n'intéresse pas les Chiliens et on le comprend aisément. La persistance du cliché qui assimile les exilés à des privilégiés, des lâches et des opportunistes est déjà plus difficile à accepter. Un travail d'éclaircissement s'impose aussi dans ce domaine afin que le cliché distillé comme un poison par les militaires eux-mêmes soit ramené à ses véritables proportions et que les Chiliens puissent voir derrière le lieu commun la réalité complexe, plurielle et souvent difficile de l'exil. À quarante ans du coup d'État, il serait temps que ce malentendu se dissipe et que les exilés soient reconnus pour ce qu'ils sont, des victimes de la dictature militaire qui méritent à ce titre une place dans ce musée. La lutte pour reconnaître les disparus (commission Rettig), puis les torturés (commission Valech), a été longue et difficile, celle pour reconnaître les exilés sera encore plus ardue, c'est pourtant la seule lutte qui reste à mener pour que cet effort de réparation s'étende à la totalité des victimes de la dictature militaire. 